\title{
Employee-Client Service Management Evaluation Based on Facial Recognition
}

\author{
Romeo E. Balcita ${ }^{1}$, Rommel E. Balcita ${ }^{2}$ and Thelma Domingo Palaoag ${ }^{3+}$ \\ 1,2 Northern Phils College for Maritime, Science and Technology, San Fernando City, La Union, Philippines \\ ${ }^{3}$ University of the Cordilleras, College Research Coordinator, Baguio City, Philippines
}

\begin{abstract}
This study focused in Service Management Evaluation (SME) for employee and client's identification of level of satisfaction in service rendered. Service evaluation plays an important role to determine the quality of service provided by an employee to their client. During the service session of the employee and client, real-time video will be automatically be analyzed by the system and evaluation result will be stored to a database. This will be used for analysis and report generation later on. The facial expression will be analyzed by the system programmed in PHP with Clmtrackr Javascript facial detection algorithm shared by collaboration projects at Github community. By embedding a database that stores evaluation reports, this can be used to create important data that will be analyzed to produce a service rating report of the employee and client's level of service satisfaction based on emotional states, particularly on their level of happiness with ratings from 0 to 10, where 0 as not happy at all and 10 as very happy. These results will help business administrators or managers in their decision making, which can lead to the success of the company. The methodology used in the study is Agile Web Engineering (AWE) approach for faster system development and System Usability Scale (SUS) for the usability test. With 65 respondents, 5 employees and 60 students, the average of the respondents' scores resulted to 75, indicating the system falls under acceptable range, grade scale is $\mathrm{C}$ and the adjective rating is good.
\end{abstract}

Keywords: facial recognition, face detection, service management evaluation, human-computer interaction, computer vision.

\section{Introduction}

Service management evaluation in any business or organization is important to the improvement of giving services, customer satisfaction and loyalty. Information technology (IT) capability to be involved in service management influences the performance of employees and the success of any organization and even in education [1]. Employee service performance usually comes from customer's perceptions through communication and behaviour. Like respect, courtesy, warmth, empathy and helpfulness. These are emotional states that a customer or employee might interact during an on-going service. This can be used to determine client's satisfaction to the service. Satisfied customers relay experience to other people, like in recommending a product or service [2]. There are two methods used in evaluating employee performance, the traditional paper-and-pen approach and the second method is an evaluation system with computers [3].

The study of facial expression was inspired by Charles Darwin that had been evolving for more than 135 years. Through face markers, individual differences in race, physical, mental and health. Humans communicate using six basic emotional states, namely: happy, surprise, fear, disgust, anger, and sad [4]. Crucial to communication and interaction to express social signals is through facial expressions [5]. Being happy signifies trustworthiness, happy faces can be trusted rather than the angry individual [6].

\footnotetext{
+ Corresponding author. Tel.: + Tel no.: 0639493666795.

E-mail address: tpalaoag@gmail.com.
} 
With Human-Computer Interaction (HCI) [7] system developers are encouraged to create systems that focus on the study of emotional information and interpreting emotions [7]. Facial landmark detection has become an important factor in face detection. Eye localization was also used as the basis of face matching which has great impact on the performance of face matching, boost face match under the required accuracy needed, and allows efficient matching without reducing performance [8]. Developed face recognition systems were found to be accurate than human in good and moderate conditions, but still humans are better during poor conditions [9]. Other factors that make face detection difficult includes: pose, orientation, facial expressions, facial sizes, luminance conditions, occlusion, structural components, gender, ethnicity, scene and complexity of image's background [10].

Facial expression reveals ample information regarding individual's emotions, intentions, condition and other states, by teaching a computer to recognize human facial expressions automatically could create a wide range of use in the future [11]. Facial expression can be used as a nonverbal communication [12]. The act of smiling positively effects on our mental status-we become happier when we laugh, like HappinessCounter [13], digital device installed in homes with single occupant for visual smile recognition.

Constrained Local Model (CLM) is a person-independent face alignment algorithm that gains high accuracy in detecting facial expression. This depends on the key features of a person's head like: the face and eyes that are tracked using a Viola-Jones type approach [14]. TurkerGaze [15] and WebGazer [16] both used the real time mechanism found in CLMtracker for tracking eye movements. CLMtrackr was used to investigate skin color to determine face detection's success [17].

This study will help decision makers have a tool in evaluating employees' performances and in relation to their clients level of satisfaction that will affect the status of the company upon success. The researchers will use the following guide questions in the development of a prototype system with CLMtrackr that shall monitor the level of happiness of client and employee during service sessions. First, what architectural framework can be adopted in developing the system? Second, what algorithm to be used in Employee-Client Service Management Evaluation Based on Facial Recognition? And third, what is the level of acceptability of the proposed study?

The objective of this research is to use two webcams on a single computer to evaluate employee-client based on facial expression - happy, together with identifying the level of satisfaction through the level of happiness of the client also based on the facial expression. Evaluation process will be easier, faster and reliable. The evaluation takes place every client's transaction.

\subsection{Conceptual Framework}

The research study is based on evaluating both the customer and employee level of happiness based on the result of a given service in real-time using facial recognition process. When an employee and client talks to each other face to face to either transact, inquire or any business matters, during their session their face make different gestures that depends on their reaction or results of the conversation. These facial gestures are emotional expressions that can be used to interpret the level of emotional states of a person. In this study, the level of happiness is concentrated in the design and development of a web-based system. The researchers will use an open source repository found in Github community for the face detection algorithm and facial detection algorithm. The emotion state monitoring and evaluation will be added to capture and compute the rating levels of the customer and employee. After a service session, the system provides the rating of the level of happiness of the customer and employee that will be used as performance evaluation rate of the employee and satisfaction rate of the customer.

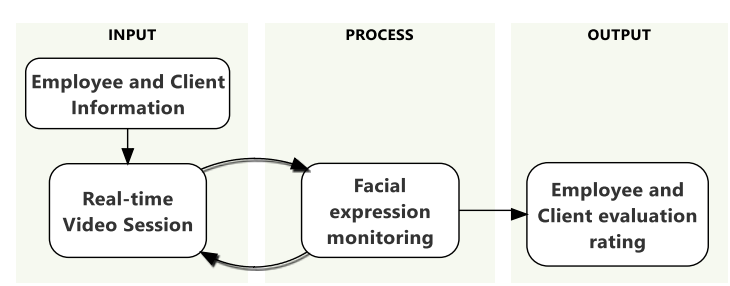

Fig. 1: IPO model. 
The IPO model shows the operational and functional concept in the design and development of the system. Before a session starts, the name of the customer and employee is entered into the system. The video session monitoring will be started when the employee activates the system, then the customer-employee service session will go on. During the session the system, three processes are involved in the evaluation, face and facial detection algorithm, and emotion state monitoring. The face detection algorithm detects the face layout, facial detection is responsible to detect the emotion state of the face, and the emotion state monitoring counts the emotional state, in this study it concentrates on happy state. The happy state will be counted up to the end of the session and will be able to store the session data into a database.

\section{Methodology}

Web development is no longer simple a new development approach is needed to cope up with the emerging advancement of system development. Our research focuses on Web Engineering approach; this requires the implementation of reusable components from standardized platforms. This method allows developers to use time efficiently by re-using codes from other systems and allows focus on the conceptual model rather than the code. In Agile web engineering (AWE) process it follows agile principles for web application development that helps tackle problems associated in the development of web based applications. The life cycle in figure 2, allows you to start web development projects as beginners or as innovations. The design and development of systems are focused more on functionality, frequent informal face-to-face communication to clients and fewer teams. AWE helps in better maintaining, implementing and continuous testing of applications, also helps in getting the solutions that will satisfy end-users.

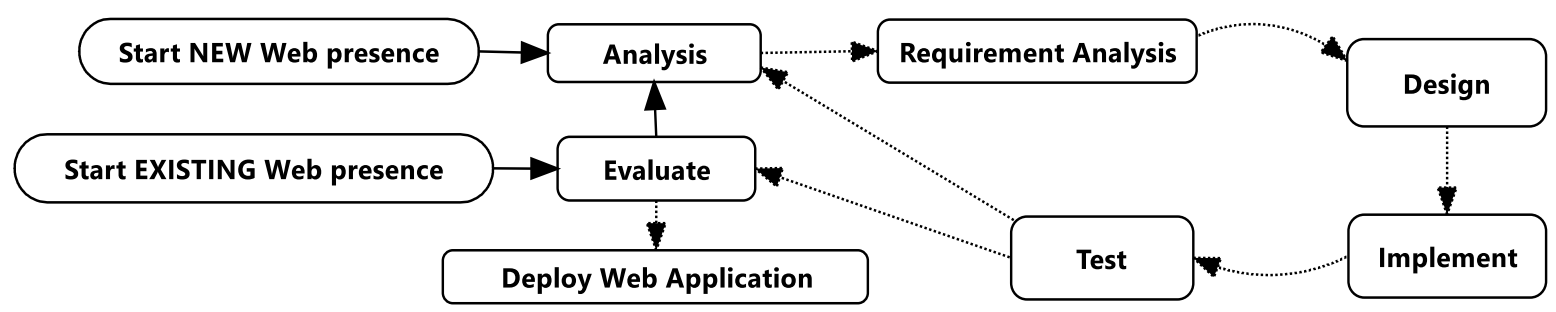

Fig. 2: AWE development cycle.

The design and development of a system to handle database and other functionality is made up of PHP and SQL, the incorporation of emotion detector from the Clmtrackr Javascript library was used to detect the emotional state of employee and client through two webcams. The system implementation and setup requires a computer with two webcams, one for the employee and the other for the client. Before any session or transaction starts, the employee is required to ask some information about the client to be the basis of the service. Then the employee may press the start button on screen to start the session, the results will be stored to a database when the employee has finished the session and stops the system.

\section{Discussion of Findings}

Architectural Framework. The architectural framework of the study shows the evaluation process cycle on either the employee or the client. The process begins when a session is started by the employee having a client. Clients being served usually took an average of fifteen minute transaction. With this time span, different facial expression can be detected that determines the level of happiness of a person. Collecting these data gives averaged results from the session reflecting the satisfaction of the client and employee.

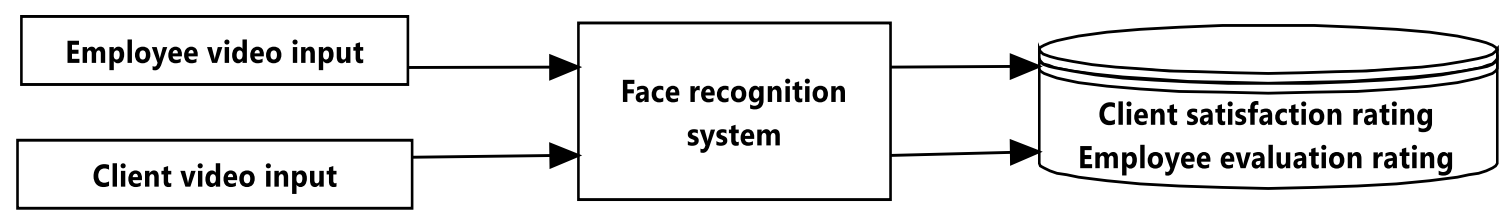

Fig. 3: Architectural framework of the study. 
Two video inputs will be needed, one directed on the employee and the other one for the client (see figure 3). The video input from the web camera will be feed to the facial recognition system getting the level of happiness of both employee and client. Ratings will be scored from 0 to 10,0 as not happy at all and 10 as very happy. The system uses Constrained Local Model (CLM) algorithm to automatically identify and recognize face from the video, then the recognized face is analyzed by Clmtrackr module where it detects, evaluate and rate the smile on the face of the person. The accumulated rates are averaged to get the final results or the needed rating of the employee and client in a particular session.

Constrained Local Model Algorithm. Constrained Local Model (CLM) works by locating sets of points in an image face. These set of points will then be connected to form facial expressions built inside the Clmtrackr Javascript mechanism. The system captures an image from the camera and detects an emotional state from the eyebrows, eyes, and mouth. The produced points in decimal values are compared giving the value for the level of happiness of the face. The accumulated values are stored in a database and will give the average rating of the employee and client for every transaction.

Evaluation Process and Result. The system is tested with 60 students and 5 employees. It uses two interfaces, one for the student and one for the employee. An employee will be evaluated and student's level of satisfaction will be rated upon pressing the Start Transaction button. Evaluation ends upon pressing the Transaction Stop button. Results are stored in a database after every transaction.
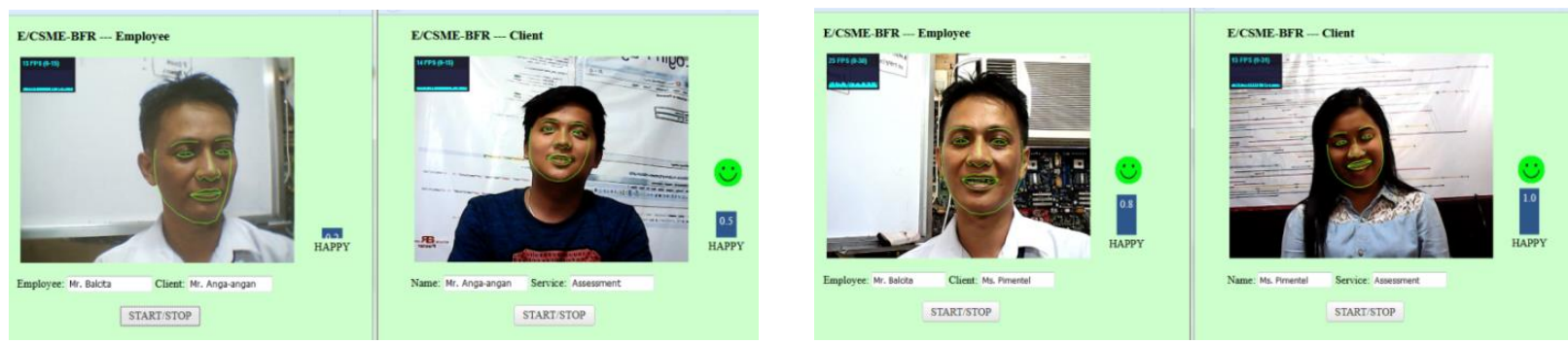

Fig. 4: Sample output 1 and sample output 2.

In figure 4, in the left side, the employee was interpreted as happy with a level of 2 while the client attained a happy level of 5. In the next figure on another instance, the employee attained a happy level of 8 and the client with a level of 10 . The system will average the ratings in every transaction and stored to a database. After the session a 10-questionnaire form from System Usability Scale (SUS) was given to evaluate the system. As a result in figure 5 shows the computed mean scores of the 65 respondents, where series 1 represents the clients and series 2 are the employees.

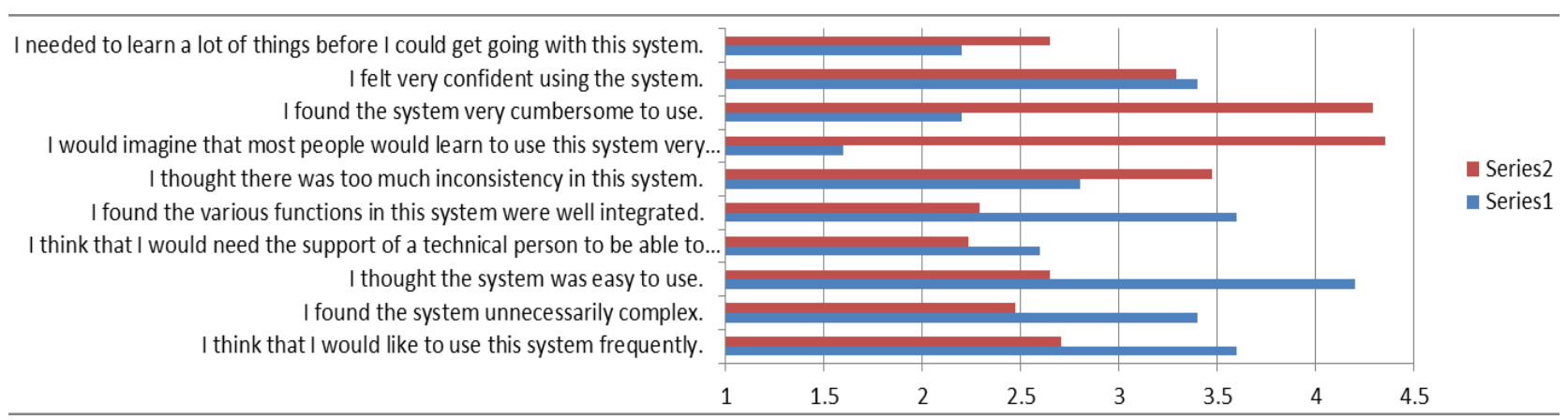

Fig. 5: Questionnaire mean scores.

Usability of the System. This research uses SUS that provides quick-and-dirty usability test [19]. The 65 respondents were given SUS 10-questionnaire to evaluate the usability of the system using the Likert scale. The clients average score is $76 \%$ and employees average score is $74 \%$, then averaging the respondents' scores resulted to $75 \%$, indicating that the system falls under acceptable range, grade scale is $\mathrm{C}$ and the adjective rating is good, see figure 6 . 


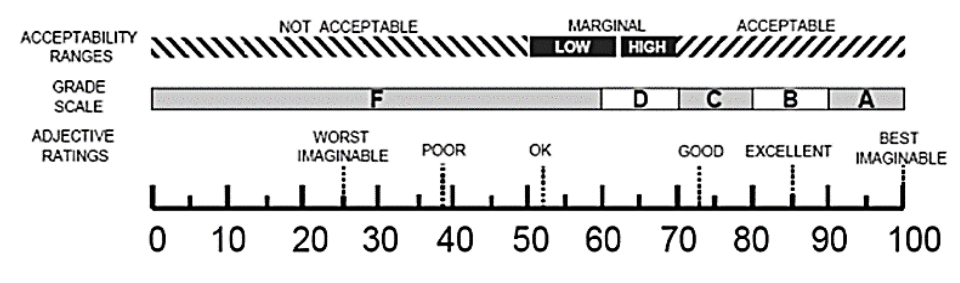

Fig. 6: SUS Interpretation.

\section{Conclusion and Recommendation}

Evaluation process needs a lot of attention because it impacts a lot in the development of a company. Making the process simple and effective thru the use of technology is a breakthrough. Video cameras are now becoming useful in our society and employee evaluation based on facial recognition is only one of its benefits. The architectural framework of this study applied in the company as one to one evaluation process with the employee and client, it suggests that the system can replace paper and pen method of evaluation. Since the CLMtrakr is an open source, it can be explored further and expand its capability in the field of facial recognition. Since in the SUS result is good it recommends the system must be improved by involving other emotional states. The system can produce more accurate results with the use of high resolution cameras and proper lighting to the evaluation area.

\section{Acknowledgements}

Clmtrackr Javascript library was made in Github by Audun Mathias Oygard in September 2017 at Oslo, Norway. We send our deepest thank you and gratitude in sharing the codes needed in this paper.

\section{References}

[1] H-T. Tsoub J-S. Chena. Performance effects of IT capability, service process innovation, and the mediating role of customer service. Journal of Engineering and Technology Management. vol. 29, issue 1, pp. 71-94. 2012.

[2] D. Gremler, A. van Riel, M. van Birgelen C. Delcourt. Effects of perceived employee emotional competence on customer satisfaction and loyalty: The mediating role of rapport. Journal of Service Management. vol. 24 issue: 1, pp.5-24. 2013.

[3] J. Gouvernel, H. Yacobovitz. Employee Evaluation System. Google Patents. US20160275431A1. 2015.

[4] D. Keltner, M. Shiota, M. O'Sullivan, M. Frank, D. Matsumoto. Handbook of emotions. New York: Guilford Press. 2008. pp. 211-234.

[5] O. Garrod, H. Yu, R. Caldara, P. Schyns, R. Jack. Facial expressions of emotion are not culturally universal. PNAS. vol 109 no. 19. pp. 7241-7244. 2012.

[6] A. Todorov, N. N. Oosterhof. Shared perceptual basis of emotional expressions and trustworthiness impressions from faces. PsycARTICLES: Journal Article. vol 9, no.1, pp. 128-133. 2009.

[7] S.K. Bandyopadhyay, M. Tech, G. Yasmin. Detecting facial expression in images. Journal for Research. vol 2, issue: 2, pp. 30-41. 2016.

[8] X. Liu, F.Su. A robust facial landmark detection method in multi-views. IEEE - Visual Communications and Image Processing (VCIP). 2015.

[9] J. Naruniec. Discrete area filters in accurate detection of faces and facial features. ScienceDirect - Image and Vision Computing. vol 32, issue 12, pp. 979-993. 2014.

[10] AJ. O'Toole, X. An, J. Dunlop, V. Natu, P.J. Phillips. Comparing face recognition algorithms to humans on challenging tasks. Journal ACM Transactions on Applied Perception (TAP), vol. 9, issue 4, no. 16. 2012.

[11] E. Cerezo, S. Baldassarri, I. Hupont. Sensing facial emotions in a continuous 2D affective space. Systems Man and Cybernetics (SMC), 2010 IEEE International Conference. 2010.

[12] J.A. Hall, T.G. Horgan, M.L. Knapp. Nonverbal Communication in Human Interaction. Cengage Learning, p. 528. 2013.

[13] J. Rekimoto, H. Tsujita. HappinessCounter: smile-encouraging appliance to increase positive mood. CHI '11 Extended Abstracts on Human Factors in Computing Systems. pp. 117-126. 2011.

[14] S.W. Chew, P. Lucey, S. Lucey, S. Luvey, J. Saragih, J. Cohnn, S. Sridharan. Person-independent Facial Expression Detection Using Constrained Local Models. Automatic Face \& Gesture Recognition and Workshops (FG 2011), 2011. 
[15] P. Xu, K. A. Ehinger, Y. Zhang, A. Finkelstein, S. R. Kulkarni, J. Xiao. TurkerGaze: Crowdsourcing Saliency with Webcam based Eye Tracking. Computer Vision and Pattern Recognition (cs.CV). 2015.

[16] A. Papoutsaki, P. Sangkloy, J. Laskey, N. Daskalova, J. Huang, J. Hays. WebGazer: Scalable Webcam Eye Tracking Using User Interactions. Proceedings of the Twenty-Fifth International Joint Conference on Artificial Intelligence - IJCAI 2016.

[17] A. Papoutsaki. Investigating the Inclusivity of Face Detection. CMC Senior Theses. 2018.

[18] J. Brooke. SUS: A Retrospective. Journal of Usability Studies. vol. 8, issue 2, pp. 29-40. 2013. 